\title{
PHYSICAL-LAYER NETWORK CODING IN OPTICAL NETWORKS
}

\author{
Calvin C K Chan and Zhixin Liu
}

\author{
Department of Information Engineering, The Chinese University of Hong Kong, Shatin, N.T., Hong \\ Kong SAR, China. ckchan@ie.cuhk.edu.hk
}

\begin{abstract}
Physical-Layer Network Coding (PNC) is a promising technique to improve the network throughput of full-duplex transmission in a wireless two-way relay channel. Here, we explore the application of PNC in optical systems and networks. Two transmitted optical signal frames are simply added together to form an optical PNC-coded composite frame, which shares the same network channel/resource. At their destined node, the composite signal is directly-detected, before being decoded by subtracting one of the two transmitted signal frames, which is either already known or stored locally, from the received $P N C$-coded composite frame.
\end{abstract}

Keywords: physical layer network coding, optical networks

\section{INTRODUCTION}

Driven by the recent popularity of high-definition videos and data-intensive applications, a steady increase of data traffic in optical networks by $40 \%$ every year has been witnessed [1]. To meet such requirement, point-to-point transmission has reached $400 \mathrm{~Gb} / \mathrm{s}$ per channel and beyond [2]. Meanwhile, more flexible networks are also required to provide various kinds of network functionalities and to assure network reliability. Recently, network coding [3,4] has aroused a lot of attention for its potential to enhance the throughput and robustness of multicast networks. It has been realized by performing linear operations, such as logic exclusive-OR (XOR), on two signals at the network layer, so as to share the same network link and reduce the required network resources. Inspired by this idea, physical-layer network coding (PNC) has been proposed to further improve the network throughput [5]. Instead of avoiding physical-layer interference, PNC exploits the interference of two signal frames so as to increase network capacity. It has attracted lots of interests in wireless communications in the past few years [6].

In the field of optical communications, the general idea of PNC has great potential to further benefit optical networks. However, the wisdom from wireless communications cannot be directly applied to optical communications, as they are quite different in physical characteristics, network structure, and system components. This opens up a new window because of its implications and new design requirements. The resolution of the many interesting and critical issues in optical physical-layer network coding (OPNC) may lead to new technology and innovations.

In [7], an all-optical XOR device for network coding was assumed and showed its benefit in a $2 \times 2$ multicast network with a shared link. In [8], Manley et al. showed the effectiveness of using network coding for dedicated protection in the optical layer. In addition to research in the network layer, the first OPNC experiment [9] has been demonstrated and characterized. Moreover, an all-optical XOR subsystem [10] has been demonstrated as a physical-layer network coding module. Recently, an OPNC system has also been demonstrated to boost the throughput in Orthogonal Frequency Division Multiplexing (OFDM) fiber-wireless network [11].

In this paper, we review the applications of OPNC in optical communications as well as the challenges and enabling technologies. Specifically, we will focus on OPNC systems in which the optical signals are simply combined in optical power, without any further all-optical signal processing. The optical signals arriving at the OPNC router are usually asynchronized.

The rest of this paper is organized as follows. Section 2 illustrates how PNC works in a two-way relay channel (TWRC) in wireless communications. Section 3 reviews the application of OPNC in multicast protection and throughput enhancement in fiber-wireless networks. Section 4 summaries the paper.

\section{PHYSICAL-LAYER NETWORK CODING}

\section{A. Principle of PNC}

The principle of PNC can be illustrated through a TWRC, as shown in Fig. 1. The TWRC is a basic network unit in which network node 1 and network node 2 wish to exchange information through a router $R$ in between. Fig. 1(a) shows the traditional scheduling scheme, while Fig. 1(b) shows the PNC scheduling scheme. In traditional scheduling scheme, interference is usually avoided by prohibiting the overlapping of signals from nodes 1 and 2 with $R$ in the same time slot. As illustrated in Fig. 1(a), node 1 first sends $S_{l}$ to $R$, and then $R$ forwards $S_{1}$ to node 2 . After that, node 2 sends $S_{2}$ in the reverse direction. A total of four time slots are needed for the exchange of two frames in opposite directions. 


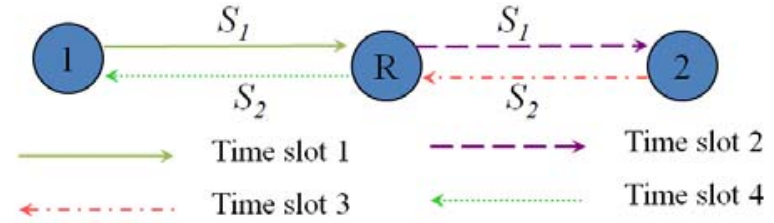

(a)

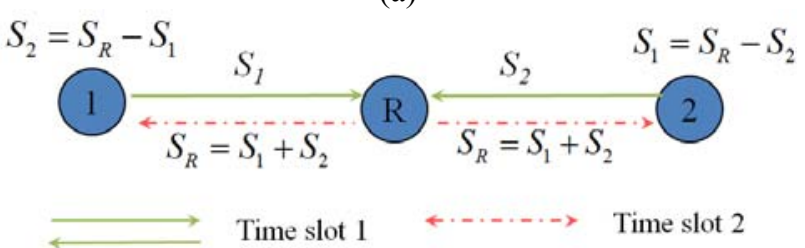

(b)

Fig. 1. (a) Traditional scheduling scheme. (b) PNC scheduling scheme.

The PNC scheme, however, exploits the interference and transmits $S_{1}$ and $S_{2}$ simultaneously to $R$ in the first time slot. Then $S_{l}$ and $S_{2}$ are encoded at $R$. The encoding can be XOR operation as used in [5] or a simple combination of electromagnetic waves, as proposed in [12]. In OPNC, we consider simple power addition operation to perform the encoding. After that, $R$ broadcasts the encoded signal $S_{R}$ to both nodes 1 and 2 in the second time slot. Nodes 1 and 2 then perform decoding operation to retrieve the transmitted information. As illustrated in Fig. 1 (b), PNC requires only two time slots for the exchange of two frames.

\section{B. Enabling Technology for OPNC}

OPNC requires proper encoding operation at the router and decoding operation at the receiver. To simplify the system and reduce the cost, encoding operation based on simple power addition is desired. However, the addition of two optical signals through a power coupler raises many issues at the receiver side, such as:

(a) How to resolve the carrier frequency offset between the interfered signals?

(b) How to recover the clock from interfered signal?

(c) How to deal with the incoherent phase noise of the two sources.

(d) How to compensate the different transmission impairments of the two signals?

The above mentioned problems are worth investigating in order to put OPNC into practice.

\section{APPLICATIONS OF OPNC IN OPTICAL COMMUNICATIONS}

A. OPNC based Multicast Protection

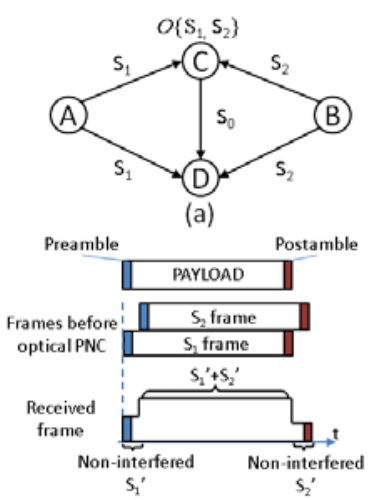

(b)

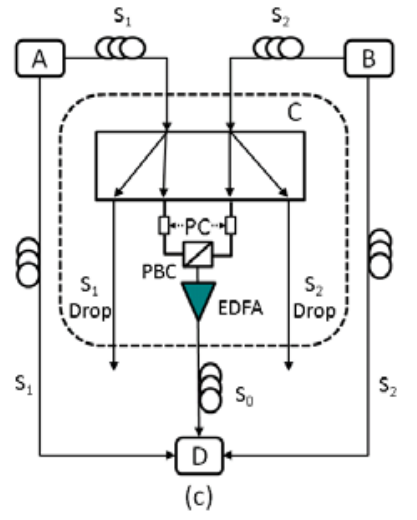

Fig. 2. (a) Example network utilizing network coding. (b) Frames before and after network coding. (c) Proposed optical PNC implementation.

The principle of multicast protection using OPNC is illustrated in Fig.2. Consider a four-node network, as depicted in Fig. 2(a), A (node A) and B (node B) simultaneously transmit their respective multicast frames, $S_{1}$ and $S_{2}$, to both $\mathrm{C}$ (node C) and D (node D). The traditional way to protect single link failure (e.g. A-D) is to set up a connection between $\mathrm{C}$ and $\mathrm{D}$, which relays $S_{l}$ from $\mathrm{C}$ to $\mathrm{D}$. However, this incurs a certain setup time, which is undesirable when the data is very sensitive to network delay. To protect links A-D and B-D against any single link failure, C needs to relay both $S_{1}$ and $S_{2}$ to D on link C-D. By applying OPNC, if $S_{1}$ and $S_{2}$ overlap in time domain, C only needs to forward a function $S_{0}=$ $O\left\{S_{1}, S_{2}\right\}$ to D. Here, $O\left\{S_{1}, S_{2}\right\}$ can be any function which has the property that $S_{2}$ can be derived from $S_{1}$ and $\mathrm{S}_{0}$ in case of failure in link B-D only, while $S_{1}$ can be derived from $S_{2}$ and $S_{0}$ in case of failure in link A-D only. As a result, the network resources for a single link failure protection are saved.

Fig. 2(b) shows the structures of the optical frames before and after OPNC. Each frame comprises both preamble and postamble, which are unique for each source node and the lengths of the two input frames are assumed to be equal. The waveform of $S_{0}$ generated through optical PNC is the addition of the waveforms and gives $S_{1}{ }^{\prime}$ and $S_{2}{ }^{\prime}$ (i.e. the respective replicas of $S_{1}$ and $S_{2}$ ) with a certain time shift between them, such that the leading and the trailing portions of $S_{0}$ are free of signal interference. Frame-level scheduling is needed to assure this.

To illustrate the process of OPNC decoding, it is assumed that the bit sequence of node 1 is known and the bit sequence of $S_{2}$ is to be decoded from $S_{0}$. From the interference-free preamble and postamble in $S_{0}$, it can be determined whether $S_{1}{ }^{\prime}$ or $S_{2}{ }^{\prime}$ comes earlier in time domain. Without loss of generality, here we assume that $S_{l}{ }^{\prime}$ comes earlier. With the interference-free preamble of $S_{l}{ }^{\prime}$ extracted from $S_{0}$, the precise timing and amplitude information of $S_{1}$ component contained in $S_{0}$ can be determined. Together with the known bit sequence of $S_{l}$, the waveform of $S_{1}{ }^{\prime}$ can be reconstructed, which is then subtracted from $S_{0}$ so as to recover $\mathrm{S}_{2}{ }^{\prime}$. Thus, the bit 
sequence of $S_{2}$ can be derived. The impulse response of the system, which is utilized for reconstructing $S_{I}{ }^{\prime}$, can be measured in advance or estimated from the interference-free preamble.

Fig. 2(c) shows the system architecture of the proposed OPNC scheme for an optical network, whose topology is the same as that in Fig. 2 (a). At node C, a copy of the input frame $S_{l}$ from node A and a copy of the input frame $S_{2}$ from node B are combined, via a polarization beam combiner (PBC), which multiplexes $S_{1}$ and $S_{2}$ on two orthogonal polarizations. Thus, the OPNC frame $S_{0}$ is generated. Polarization controllers (PCs) are used to adjust the polarizations of the input frames so that the insertion loss at $\mathrm{PBC}$ can be minimized. The insertion loss at PBC can be monitored by measuring the output optical signal power at the other output port of the PBC. Time shifting between the frames is realized by proper scheduling in the network layer. The network-coded signal $S_{0}$ is then amplified by an erbium-doped fiber amplifier (EDFA) before being relayed to node $\mathrm{D}$, where the network-coded signal is detected by a single PD and the missing frame can be recovered, when necessary.

\section{B. OPNC for improving transmission efficiency}

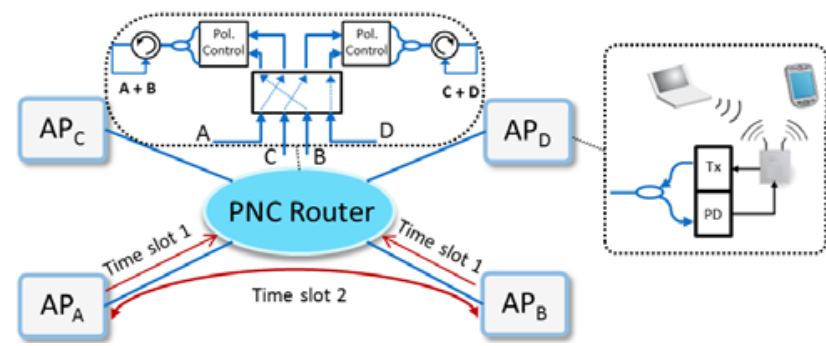

(a)

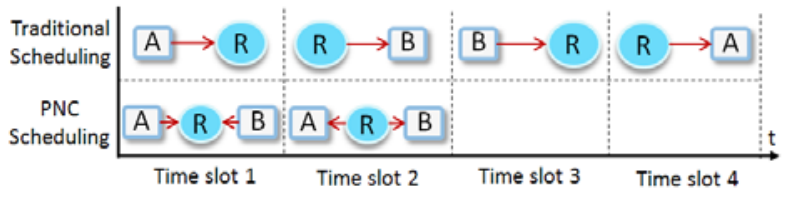

(b)

Fig. 3. (a) Proposed system architecture. (b) Comparison of the scheduling approach

Another important application of OPNC is to increase the network transmission capacity. As mentioned before, TWRC is a basic building block of network. Therefore, in the second scenario, we exploit OPNC's potential in increasing the transmission capacity in hybrid fiber-wireless (FiWi) network that can be decomposed into multiple TWRCs. In this work, we incorporate the key concept of OPNC in a FiWi network [11]. Unlike other demonstrations, our current prototype [12]. adopts the OFDM frame format, as specified in the widely adopted $802.11 \mathrm{n}$ WLAN standards. Notably, our OFDM OPNC scheme does not require symbol-level synchronization of the OFDM packets, and only requires moderate modifications of the packet preamble in 802.11 OFDM physical layer specifications (PHY).

Consider a four-node network as depicted in Fig. 3(a). Four access points (APs) are connected through a single router in a star topology. Suppose that node $\mathrm{A}\left(A P_{A}\right)$ and node $\mathrm{B}\left(A P_{B}\right)$ want to exchange two packets with each other. Fig. 3(b) compares traditional scheduling and PNC scheduling for this purpose. Similar to the PNC principle discussed in section 2, in traditional scheduling, node A sends its packet $P_{A}$ to the PNC router in the first time slot. The PNC router then forwards it to node B in the second timeslot. Two more time slots are needed for the delivery of packet $P_{B}$ from node $\mathrm{B}$ to node $\mathrm{A}$ in a similar fashion. A total of four timeslots are needed. Nevertheless, OPNC scheduling can achieve the same goal with only two timeslots. In the first time slot, nodes $\mathrm{A}$ and $\mathrm{B}$ transmit their packets simultaneously to the PNC router. Let $S_{A}$ and $S_{B}$ denote the signals of packets $P_{A}$ and $P_{B}$, respectively. These signals are superimposed on each other to form $y_{R}=S_{A}+S_{B}$, that is the arithmetic sum of the two signals. The router then broadcasts $y_{R}$ to both nodes $\mathrm{A}$ and $\mathrm{B}$ in the second timeslot. After receiving $y_{R}$, node A then extracts $S_{B}$ by subtracting its locally pre-stored information $S_{A}$ from $y_{R}$. Node B obtains $S_{A}$ in a similar fashion. Note that no additional physical-layer processing other than simple optical power addition is performed at the optical PNC router. Hence, the complexity of the optical PNC router is kept low while the throughput is doubled.

At the PNC router, an optical switch is used to form multiple TWRCs, each with two end nodes exchanging information. To realize the addition operation in a direct-detection optical system, two polarization controllers are employed to control the signal polarization, so that the signals from nodes $\mathrm{A}$ and $\mathrm{B}$ are in orthogonal polarization before being power-combined via an optical coupler. An optical circulator is used to loop back the composite signal for broadcast back to both nodes $\mathrm{A}$ and $\mathrm{B}$. In a star-topology network with $N$ nodes, N/2 TWRCs can be formed at the same time. PNC doubles the throughput of each TWRC. Thus, the total network throughput can be doubled with paired traffic patterns.

\section{SUMMARY}

The applications of PNC to optical communications have been explored and OPNC schemes have been reviewed. In general, they do not require bit synchronization and it is tolerant to power imbalance between the two input optical frames before PNC. One of the important applications of OPNC is to effectively implement multicast protection in optical networks. We have also demonstrated the first OFDM-OPNC implementation in a star-topology FiWi system. The OFDM OPNC scheme doubles the system throughtput. Tight symbol-level synchronization is not needed thanks to the use of OFDM. 


\section{ACKNOWLEDGMENTS}

The project was supported by AoE Grant E-02/08, and GRF 414911 from Hong Kong RGC.

\section{REFERENCES}

[1] Cisco visual networking index: Forecast and methodology. 2009 - 2015. [Online]. Available: www.cisco.com.

[2] Q. Yang, A. A. Amin, X. Chen, Y. Ma, S. Chen, and W. Shieh "428-Gb/s single-channel coherent optical OFDM transmission over $960-\mathrm{km}$ SSMF with constellation expansion and LDPC coding" Optics Express, Vol. 18, Issue 16, pp. 16883-16889 (2010).

[3] R. Ahlswede, N. Cai, S-Y. R. Li and R. W. Yeung, "Network information flow," IEEE Trans. Information Theory, 46, 1204-1216 (2000)

[4] S-Y. R. Li, R. W. Yeung, and N. Cai, "Linear network coding," IEEE Trans. Information Theory, 49(2), 371-381 (2003)

[5] S. Zhang, S.-C. Liew, and P. Lam, "Hot topic: Physical-layer network coding," in Proc. 12th Ann. ACM Int. Conf. Mobile Computing and Netw. (MobiCom 2006), Los Angeles, CA, Sep. 2006.

[6] L. Lu and S. C. Liew, "Asynchronous Physical-Layer Network Coding", IEEE Trans. on Wireless Commun., vol. 11, no. 2, pp. 819-831, Feb. 2012.

[7] R. Menendez and J. Gannett, "Efficient, fault-tolerant all-optical multicast networks via network coding," in Optical Fiber Communication Conference (OFC/NFOEC), 2008, pp. 1-3, San Diego, CA USA, 2008.

[8] E. D. Manley, J. S. Deogun, L. Xu, and D. R. Alexander, "All-optical network coding," IEEE/OSA Journal of Optical Communications and Networking, vol. 2, no. 4, pp. 175 -191, Apr 2010.

[9] Z. Liu, M. Li, L. Lu, C.-K. Chan, S.-C. Liew, and L.-K. Chen, "Optical physical-layer network coding," IEEE Photonics Technology Letters, vol. 24, no. 16, pp. 1424 -1427, Aug. 2012.

[10] Y. An, F. D. Ros and C. Peucheret, "All-optical network coding for DPSK signals," in IEEE/OSA Optical Fiber Communication Conference (OFC/NFOEC), paper JW2A, Anaheim, 2013.

[11] S. Katti, S. Gollakota, and D. Katabi, "Embracing wireless interference: Analog Network Coding," in ACM SIGCOMM, Kyoto, Japan, 2007.

[12] Z. X. Liu, L. Lu, L. Z. You, C. K. Chan, S. C. Liew, "Optical physical-layer network coding over fiber-wireless," European Conference on Optical Communications, (ECOC), Paper Mo.3.F.3, UK, London, 2013. 